Birgit Panke-Kochinke (PD Dr.), Gabriele Krause (MSc), Olga Klimann (MSc)

Deutsches Zentrum für neurodegenerative Erkrankungen (DZNE), Witten

\title{
Ein wissenschaftlicher Diskurs über Demenz - Erste Ergebnisse der exemplarischen Anwendung eines integrativen Analyseansatzes
}

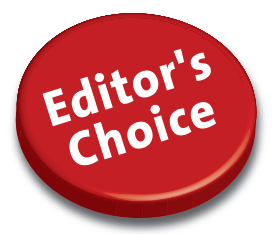

- Was ist (zu dieser Thematik) schon bekannt?

Der Begriff der Demenz ist im wissenschaftlichen Diskurs nicht einheitlich bestimmt.

Was ist neu?

Die Betonung der starken Belastung von Angehörigen von Menschen mit Demenz führt zu Versorgungsstrukturen, die eher auf deren Bedürfnisse und weniger auf die von Menschen mit Demenz selbst ausgerichtet sind.

- Welche Konsequenzen haben die Ergebnisse für die Pflegepraxis? Der Mensch mit Demenz selbst sollte mehr in den Blick der Forschung gelangen. Dessen Belastungen, aber auch Ressourcen gilt es vermehrt wahrzunehmen, um diese Zielgruppe angemessen unterstützen zu können.

Hintergrund: Eine Analyse des wissenschaftlichen Diskurses zum Umgang von Menschen mit Demenz innerhalb der Pflege- und Gesundheitsforschung in Deutschland fehlt bisher.

Fragestellung: Wie werden Menschen mit Demenz im pflege- und gesundheitswissenschaftlichen Diskurs dargestellt und welche Perspektiven lassen sich daraus ableiten?

Methode: Mithilfe eines neu entwickelten integrativen Analyseverfahrens wird ein Diskurs in der Analyse des Umgangs mit Menschen mit Demenz im wissenschaftlichen Diskurs in der Pflege- und Gesundheitsforschung rekonstruiert.

Ergebnisse: Inhaltlich wird Demenz im exemplarisch rekonstruierten wissenschaftlichen Diskurs vor allem unter dem Aspekt einer Belastung für Angehörige diskutiert. Sie werden als diejenigen bezeichnet, denen durch die

Manuskript eingereicht am 7.4.2014 Manuskript akzeptiert am 24.10.2014
Menschen mit Demenzeine große Belastung entsteht.

Schlussfolgerungen: Ein methodisches Verfahren der integrativen Analyse konnte durch den vorgestellten Forschungszugang erprobt werden. Zukünftig ist die Perspektive des Menschen mit Demenz im Forschungskontext stärker zu berücksichtigen.

Schlüsselwörter: Diskurs, Demenz, integrative Analyse, Schlüsselkonzepte, Vermittlungsvariablen

Einleitung und Fragestellung

Foucault konnte die Demenz in seiner Geschichte des Wahnsinns und seiner Funktionsbestimmung in der Gesellschaft im 17. und 18. Jahrhundert noch als eine Art unspezifisches Sammelbecken für alles das identifizieren, was mit Unvernunft bezeichnet wurde (Foucault, 1969; 256-267). Der Begriff der Demenz hatte seines Erachtens im damaligen wissenschaftlichen Diskurs der Medizin die Funktion, eine krankheitsbedingte Unvernunft zu bezeichnen. Sie erschien in ihrem Kern unbestimmt, in ihrem Sitz, dem Gehirn, und ihren Ursachen, einer Störung der Nervenfasern in der Zuleitung zu eben diesem Gehirn, bestimmbar. Die Unbestimmbarkeit des Begriffes der Demenz bzw. der krankheitsbedingten Unvernunft stand in seiner Analyse demnach für ihre Wirksamkeit. Doch was versteht der aktuelle wissenschaftliche Diskurs zu Beginn des 21. Jahrhunderts unter Demenz?

Die typische Herangehensweise an die Beantwortung dieser Frage würde in einem ersten Schritt eine umfangreiche und systematische Literaturrecherche erfordern. Methodische Verfahren der Diskursanalyse, wie sie aktuell in einer großen Spannbreite existieren (Wrana, Ziem, Reisig, Nonhoff \& Angermüller, 2014), wären des Weiteren daraufhin zu überprüfen, ob sie für einen solchen Zugang geeignet erscheinen. Die einzelnen Diskursfelder wären exakt zu bestimmen, einzugrenzen und zu analysieren. Die im Folgenden referierten Ergebnisse greifen aus diesem umfangreichen Diskursprojekt einen für die wissenschaftliche Diskussion wichtigen inhaltlichen und einen methodischen Teilaspekt auf.

Der inhaltliche Aspekt: Man kann begründet davon ausgehen, dass eines der zentralen Leitsymptome der Krankheitsbilder der Demenz im wissenschaftlichen Diskurs der Gegenwart die sukzessiv voranschreitende Einschränkung von kognitiven Fähigkeiten ist. Dadurch bleibt die Frage nach der krankheitsbedingten Unvernunft, die Foucault für den medizinischen Diskurs des 17. und 18. Jahrhunderts erfasst hat, zumindest virulent. Ob Demenz dabei noch als unspezifisches Sammelbecken für Unvernunft zu bezeichnen ist, müsste, wie angedeu- 
tet, zunächst einmal für die verschiedenen Diskursfelder analysiert werden. Das ist nur im Rahmen eines größeren Untersuchungsprojektes möglich. Es ist jedoch möglich, an einem exemplarisch ausgewählten und methodisch definierten Diskurs anzusetzen und sich zunächst einmal die Frage zu stellen, wie die Gesellschaft mit Menschen umgeht, auf die das Krankheitsbild der Demenz angewendet wird. Die Rekonstruktion des wissenschaftlichen Diskurses der Pflege- und Gesundheitsforschung in Deutschland über den Umgang mit Menschen mit Demenz beschreibt dabei den formalen Rahmen des analysierten Diskurses. Die zentrale Frage lautet, wie Menschen mit Demenz im pflege- und gesundheitswissenschaftlichen Diskurs dargestellt werden, und welche Perspektiven sich daraus ableiten lassen.

Der methodische Aspekt: Einen Diskurs zu analysieren bedeutet nach Foucault, die zentralen Bereiche, in denen über ein Thema gesprochen wird, zu erfassen (Foucault, 2009; 213). Und genau an diesem Punkt tritt ein Problem auf, das einer methodischen Überlegung bedarf: Die Massenproduktion und Verbreitung von Texten, die in der Gegenwart durch die Medien möglich ist und auch wahrgenommen wird, ist gegenüber den vorherigen Jahrhunderten um ein Vielfaches gestiegen. Es stellt sich vor diesem Hintergrund die methodisch zu beantwortende Frage, wie diese Textmassen angemessen berücksichtigt werden können. Dafür wird ein integrativer Analyseansatz entwickelt und erprobt.

Der Diskursbegriff nach Foucault wird als Ausgangspunkt des im Folgenden realisierten integrativen Analyseansatzes gewählt. Das methodische Vorgehen, insbesondere die methodologischen Überlegungen schließen an den Begriff eines Untersuchungsprogramms an und bieten somit die Option, in der Rekonstruktion der Argu- mentationsmuster eine Logik der Strategie (Foucault, 2009; 353) zu erfassen. Zur Realisierung dieses Vorhabens wird ein sechsschrittiges Verfahren verwendet, in seinen Grundzügen präsentiert und exemplarisch an dem gewählten eingegrenzten Diskurs erprobt. Die Ergebnisse der mithilfe der Suchstrategie (Schritt 1 bis 5) erfassten regelgeleiteten Textauswahl werden in Schritt 6 (s.u.) des methodischen Vorgehens als zwei einander ergänzende Zugangsweisen präsentiert. Die Diskussion der Ergebnisse wird unter einer inhaltlichen und methodischen Perspektive vorgenommen. Abschließend werden Perspektiven für die Pflege- und Gesundheitswissenschaft formuliert.

\section{Der Diskursbegriff nach Foucault}

Nach Foucault bezeichnet ein Diskurs «die Gesamtheit erzwungener und erzwingender Bedeutungen, die die gesellschaftlichen Verhältnisse durchziehen» (Foucault, 2009; 213). Es handelt sich um ein strategisches Feld, das zu einem Ort oder auch Instrument der Konfrontation werden kann (Foucault, 2009; 213). Er ist eine soziale Tatsache und als solche hat er eine Machtstruktur. Er ist selbst «ein Element in einem strategischen Dispositiv aus Machtbeziehungen» (Foucault, 2009; 221). Ihn interessiert am Problem des Diskurses nicht der Sinn einer Aussage, sondern die Funktion, «die man der Tatsache zuweisen kann, dass dies zu diesem Zeitpunkt gesagt worden ist. Das nenne ich Ereignis. Mir geht es darum, den Diskurs als eine Folge von Ereignissen zu betrachten» (Foucault, 2009; 223).

Dieser Begriff von Diskurs impliziert nun nach Foucault eine Abgrenzung gegenüber einer Beschreibung phänomenologischen Typs oder anderer interpretativer Methoden. «Ich suche nicht hinter dem Diskurs nach einer
Macht, die dessen Quelle sein soll, wie es in der Beschreibung phänomenologischen Typs oder bei jeder andern interpretativen Methode geschieht. Ich gehe vom Diskurs als solchem aus. In einer phänomenologischen Beschreibung möchte man aus dem Diskurs etwas über das Subjekt des Diskurses ableiten. Man versucht, im Diskurs etwas über die Intentionen des sprechenden Subjekts zu erfahren - ein Denken, das sich gerade vollzieht» (Foucault, 2009; 221).

Als regelgeleitete Praktik gliedert sich für Bohnsack, Marotzki und Meuser (2003) ein Diskurs in vier diskursive Formationen: die Formation der

- Gegenstände,

- Äußerungsmodalitäten,

- Begriffe und

- Strategien.

«Die diskursiven Formationen erzeugen die Gegenstände, die sie behandeln; sie bestimmen den Gebrauch und das semantische Feld der Begriffe, die zur Beschreibung dieser Gegenstände verwendet werden; sie legen die Modalitäten fest, in denen eine Äußerung legitimer Weise erfolgen kann; schließlich entscheiden sie über die möglichen Strategien, die die Diskursteilnehmer mit ihrer Rede verfolgen können. Ihr Zusammenspiel produziert spezifische Diskurse und bestimmt die operativen Regeln, denen diese Diskurse gehorchen» (Bohnsack et al., 2003; 36 - 37).

Letztendlich sind es diese diskursiven Formationen, die als regelgeleitete Praktiken das Feld der Macht nicht nur beschreiben, sondern darauf im Prozess auch zurückwirken und es mitbestimmen. Wenn der Gegenstand Demenz z.B. als eine Krankheit beschrieben wird, die durch eine kognitive Beeinträchtigung und Verhaltensauffälligkeiten und damit auch den Verlust von Grundlagen des Vernunftbegriffes gekennzeichnet ist, handelt es sich um Begriffe, die in den dazugehörigen 
Kontexten dazu beitragen, das Handeln sowohl von Menschen, die von Demenz betroffen sind, als auch das derjenigen Personen, die in pflegerischen Kontexten arbeiten, im Sinne dieser Verortung zu beeinflussen. Beide Personengruppen richten demnach ihre Handlungspraxis unbewusst danach aus. D.h. Handlungsstrategien konstruieren sich aus der Art und Weise, wie der Gegenstand definiert wird und tragen damit gleichzeitig zur Rekonstruktion und der strategischen Formation des Diskurses bei. Die am Diskurs Beteiligten nutzen die Begriffe, entfalten ihre Äußerungsmodalitäten innerhalb dieses Regelwerks und handeln entsprechend. Sich über Demenz zu äußern, mit Demenz zu leben und in einem pflegerischen Handlungsfeld tätig zu sein bedeutet, dass man sich der Macht dieses Diskurses, diesem Foucaultschen Denkmodell folgend, praktisch nicht entziehen kann.

\section{Das methodische Vorgehen}

\section{Methodologische Vorüberlegungen}

Einen Diskurs zu rekonstruieren bedeutet nun keineswegs, ein einheitliches methodisches Verfahren anzuwenden. Es ist eher ein Untersuchungsprogramm (Bohnsack et al., 2003; 38), das den Rahmen für die Entwicklung eines solchen methodischen Verfahrens bildet. Dem Gegenstand der Analyse, dem Diskurs, ist die Methode zur Analyse seiner Struktur inhärent (Foucault, 2009; 359).

Foucault nennt seine Methoden dann auch eine Werkzeugkiste, die dazu dient, die Logik des Unbewussten durch eine Logik der Strategie zu ersetzen (Foucault, 2009; 353). Eine Diskursanalyse durchzuführen, bedeutet nach Foucault vor allem auch, die zentralen Bereiche, in denen über ein Thema gesprochen wird, die also an die Öffent- lichkeit treten, zu erfassen: das Archiv zu erschließen (Foucault, 2009; 350). «Unter dem Archiv verstehe ich zunächst alle die in einer Kultur gesagten Dinge, die aufbewahrt und verändert worden sind. Kurz, diese ganze sprachliche Masse, welche die Menschen hervorgebracht und in ihre Techniken und Institutionen gesteckt haben und die mit ihrem Dasein wie auch ihrer Geschichte verwoben sind» (Foucault, 2009; 350).

Und genau an diesem Punkt tritt ein Problem auf, das einer methodischen Überlegung bedarf: Vor dem Hintergrund der Massenproduktion und Verbreitung einer nahezu unüberschaubaren Anzahl von Texten, die in der Gegenwart vor allem durch die Neuen Medien möglich sind und auch wahrgenommen werden, muss sich die Frage der Erschließung dieses Archivs neu stellen. Es geht dabei weniger um eine lückenlose quantitative Erfassung und inhaltliche Auswertung aller zeitgenössischen Texte und Dokumente, die den Diskurs zum Thema Demenz bestimmen, sondern um eine methodisch begründete Möglichkeit, die Logik der Argumentation in ihrer strategischen Bedeutung für die Machtstruktur der Gesellschaft zu erfassen. Ein wesentlicher Ansatzpunkt dafür liegt in dem methodischen Scharnier der «Vermittlungsvariablen», das einen qualitativhermeneutischen Zugriff mit einer eher quantitativ orientierten Suchstrategie verbindet.

Diese Grundidee lässt sich methodologisch folgendermaßen begründen: Das qualitative Methodenrepertoire des rekonstruktiven hermeneutischen Zugriffs auf einen gesellschaftlich wirksamen Diskurs arbeitet mit dem theoriegestützten Erkenntnispotenzial der semantischen Struktur von Textmaterial. Wenn immanent und exemplarisch herausarbeitet werden kann, worüber in einem auch zufällig ausgewählten Text gesprochen wird, lässt sich aus einer methodologischen Perspektive heraus analytisch feststellen, ob und wenn ja welche allgemeine gesellschaftliche Relevanz dem ausgewählten Text innewohnt. So lassen sich für jeden exemplarisch ausgewählten Text zentrale kontextbezogene Inhaltskategorien und Vermittlungsvariablen erfassen. Sie bilden die Grundlage für die Erarbeitung von Argumentationsmustern, die nach Foucault auch als Logik der Strategie (Foucault, 2009; 353) bezeichnet werden können. Vor dem Hintergrund dieser generellen methodologischen Überlegungen lässt sich ein methodisches Verfahren der Umsetzung in der Form eines integrativen methodischen Zugriffs (Kelle, 2008) anwenden.

\section{Das inhaltsanalytische Analyseverfahren} Der Gegenstand jeder Art von Inhaltsanalyse ist nach Meuser «prinzipiell jede Art symbolischen Materials, seien es Texte, Bilder, Filme, Tondokumente» (zit. nach Bohnsack et al., 2003; 89). Für Mayring geht es in einer qualitativen Inhaltsanalyse dann insbesondere darum, diese im Material fixierte Koтmunikation zu analysieren, dabei theorieund regelgeleitet und damit auch systematisch vorzugehen mit dem Ziel, «Rückschlüsse auf bestimmte Aspekte der Kommunikation zu ziehen» (Mayring, 2007; 13). Generell sind dabei für ihn sowohl qualitative als auch quantitative Analyseschritte denkbar.

Um zu einer sukzessiven Verdichtung des Materials zu kommen, entwickelt er ein mehrschrittiges Ablaufschema (Mayring, 2007; 43). Im Mittelpunkt steht dabei eine Form der deduktiven oder induktiven Kategorienbildung. Generell ist für ihn die Integration qualitativer und quantitativer Analyseschritte nicht nur möglich, sondern auch je nach Fragestellung auch sinnvoll (Mayring, 2007; 45).

Sein Prozessmodell induktiver Kategorienbildung (Mayring, 2007; 75, 
Abb. 11a) wurde von Kuckartz ergänzt. Aber auch dieses Modell einer gewissermaßen «hermeneutisch-interpretativ informierte[n] Inhaltsanalyse» (Kuckartz, 2012; 5) hebt nicht die von Meuser gegenüber Mayring formulierte Problematik auf, dass es sich auch bei diesem Rahmenmodell für eine induktive Kategorienbildung, das unter dem Label der qualitativen Konnotation der Forschungsrichtung auftritt, im Prinzip eher um Erhebungs- und Auswertungsverfahren im Rahmen standardisierter Methoden und deduktivnomologische Methodologie handle (Bohnsack et al., 2003; 90). Dieser kritische Einwand war leitend für die Autorinnen, um aus dem methodischen Bezugssystem einer qualitativen empirischen Sozialforschung eine Form der induktiven Kategorienbildung zu wählen, die sich eindeutig im Feld der hermeneutischen Interpretation ansiedelt.

Die Erfassung regelgeleiteter Praktiken bedient sich also eines methodischen Verfahrens, das in der Kombination mit einem modifizierten Rahmenmodell für eine qualitative Inhaltsanalyse nach Mayring und
Kuckartz in der Lage ist, eine Vielzahl von Textdokumenten in einem vertretbaren zeitlichen Rahmen zu bearbeiten. Dafür wurde ein sechsschrittiges Verfahren angewendet (Abb. 1). Der erste bis vierte Schritt dient der Erfassung von zentralen Vermittlungsvariablen. Der Begriff der Vermittlungsvariablen bezeichnet dabei die Begriffskombinationen, die im Zusammenhang mit dem Thema Demenz in vier exemplarisch ausgewählten Texten auf der Grundlage immanenter Kategorienbildung genannt werden. In einem fünften und sechsten Schritt werden zentrale Argumentationsmuster erfasst. Sie lassen sich über Matrices erschließen (vgl. Schritt 3) und bilden die Grundlage für eine erneute Textauswahl, die als systematische Suchstrategie auf große Textmengen angewendet werden kann. Zentrale Argumentationsmuster sind kontextbezogene inhaltliche Zusammenhänge, die sich unter Vorgabe der identifizierten Vermittlungsvariablen herausarbeiten lassen. Sie ermöglichen es, diskursive Praktiken zu erschließen. Im Folgenden werden diese Schritte in der Analyse des Dis-

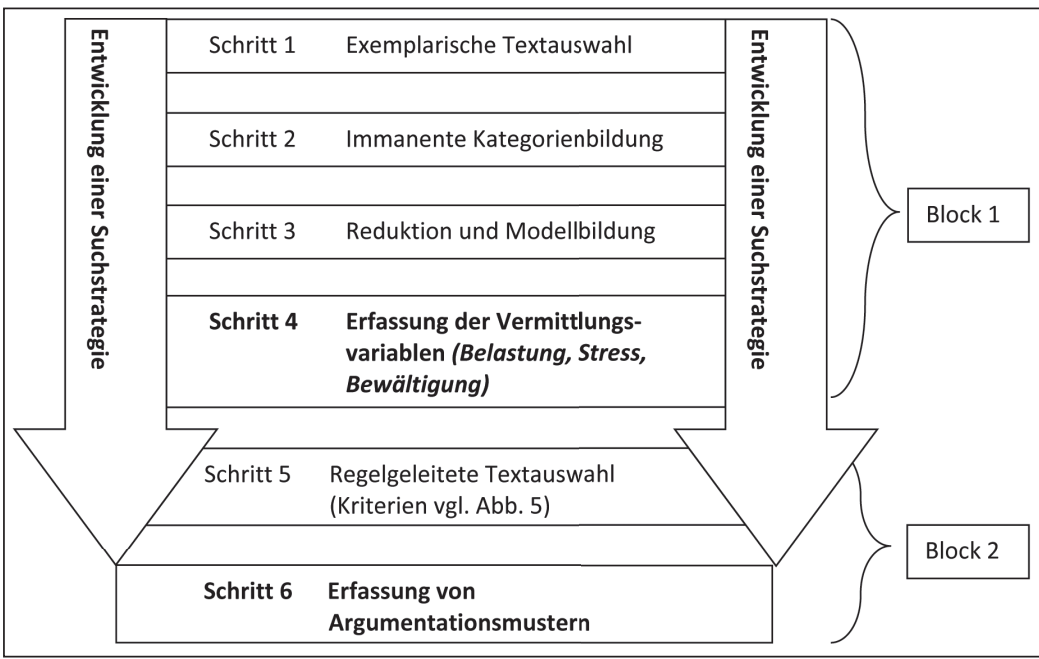

Abbildung 1: Integrativer Analyseansatz zur Verortung der Demenz in der Pflege- und Gesundheitsforschung: Methodisches Vorgehen. kurses zum Thema Demenz exemplarisch durchgeführt (Abb. 1).

\section{Exemplarische Textauswahl (Schritt 1)}

In einem ersten Schritt der Erprobung des integrativen Analyseansatzes wurde sowohl eine formale als auch inhaltliche Begrenzung vorgenommen. Vier wissenschaftliche respektive populärwissenschaftliche Texte $^{1}$ wurden aus einer im Vorfeld vorgenommenen Literaturrecherche ausgewählt. Die formale und inhaltliche Vorgabe der systematischen Zufallsauswahl war folgende: Es sollte sich um wissenschaftliche beziehungsweise populärwissenschaftliche Texte handeln, die sich auf der Grundlage von Selbstäußerungen von Menschen mit Demenz (im frühen und mittleren Stadium) mit der Analyse der Funktion und Bewältigung dieser Krankheit auseinandersetzten. Zudem sollte es sich um Zeitschriftenartikel handeln, die in ihrem Erscheinungsdatum einen Zeitraum von 1995 bis 2011 umfassen. Diese Vorgabe war formuliert worden, weil vor dem Hintergrund der vorab durchgeführten unsystematischen Literaturanalyse die begründete Vermutung bestand, dass sich eine wissenschaftliche Auseinandersetzung mit diesem Thema erst seit dem Ende der 1980er Jahre in einem größeren Umfang erkennen lässt.

\footnotetext{
1 Ausgewählt wurden folgende Texte. Sie werden in der Reihenfolge ihres Erscheinungsdatums aufgeführt. Text 1: Bender, M. P.; Cheston, R. (1997): Inhabitants of a Lost Kingdom: A Model of the Subjective Experiences of Dementia. Aging and Society, 17 (5), 513 - 532. Text 2: Menne, H. L.; Kinney, J. M.; Morhardt, D. J. (2002): «Trying to continue to do as much as they can dor. Theoretical insights regarding continuity and meaning making in the face of dementia. Dementia, 1 (3), 367 - 382. Text 3: Harman, G.; Clare, L. (2006): Illness Representations and Lived Experience in Early-Stage Dementia. Qualitative Health Research, 16 (4), 484-502. Text 4: Dechant, R.; Zimmermann, C.; Merlin, H. (2010): «Ich bin ja noch ich». Rita Dechant, Christian Zimmermann und Helen Merlin über ihren Alltag als Demenzkranke, ihre Strategie beim Outing und ihren Umgang mit dem allmählichen Verlust der Selbständigkeit. Spiegel
} Wissen, (1) 34-41. 
Der Einbezug auch englischsprachiger Texte hatte die Funktion, die Erfassung von Vermittlungsvariablen (Schritt 4 der Analyse) auf eine möglichst breite, nicht nur auf Deutschland bezogene Vergleichsebene zu stellen. Das Risiko, damit Kontexte und Vermittlungsvariablen $\mathrm{zu}$ erfassen, die sich in dem deutschsprachigen Diskurs zum Thema Demenz nicht wiederfinden ließen, wurde vor dem Hintergrund der Kenntnis der Sekundärliteratur für gering erachtet.

Immanente Kategorienbildung (Schritt 2) Die vier zufällig aus einer Gesamtzahl von 37 ausgewählten Texte wurden mithilfe von MAXQDA (Version11) zur Analyse qualitativer Daten (Kuckartz, 2007) einer immanenten Kategorienbildung unterzogen. Die in den Texten selbst verwendeten Begrifflichkeiten in ihren Kontextbezügen wurden dabei weitestgehend beibehalten. Dies führte in einem zweiten Schritt des Verfahrens dazu, dass eine der beiden CoAutorinnen zwischen 150 und 180 einzelne Kategorien pro Text bildete (siehe Abb. 1).

Reduktion und Modellbildung (Schritt 3) Um die Vielzahl der identifizierten Kategorien zu reduzieren und handhabbar zu machen, wurde anschließend in der Dreiergruppe des Autorinnenteams ein Vergleich der Kategorienbildung durchgeführt und eine formale Grenze in der Bildung von jeweils zwanzig übergeordneten Kategorien pro Text gesetzt. Eine der CoAutorinnen hatte die Texte selbst analysiert, die andere kannte die Ausgangstexte nicht. Diese Konstellation wurde gewählt, um überprüfen zu können, ob sich die Reduktion der immanent entwickelten Kategorien auch unabhängig von der Kenntnis des Ausgangstextes als tragfähig für die Erstellung von übergeordneten Kategorien eignet.
Es wurde erkennbar, dass die übergeordneten Kategorien nur geringe Abweichungen aufwiesen. Die jeweils von den beiden Co-Autorinnen gewählten eigenen Begrifflichkeiten unterschieden sich im Hinblick auf die Blickrichtung: ein eher analytischer Außen- und ein rekonstruierender Innenblick ließen sich in der gemeinsamen Diskussion zu einer übergeordneten Kategorie verbinden. Die Person, die selbst die Kodierung vorgenommen hatte, wählte dabei eher den innerperspektivischen Blick. Ihre Kategorien blieben dem Prinzip der Immanenz verbunden. Die Person, die den Text nicht kannte, neigte eher dazu, nach Strukturkriterien zu systematisieren. Die Aufgabe der dritten, das Projekt leitenden Erstautorin war es, gemeinsam mit den beiden Co-Autorinnen die Abgleichungen vorzunehmen, und vor dem Hintergrund des jeweils unterschiedlichen Wissensstandes der Beteiligten gemeinsam das in dem Text erscheinende Strukturmuster zu entschlüsseln. Jede Kombination, die gedanklich erprobt und aufgrund der Kategorien sinnvoll erschien, wurde daraufhin überprüft, ob sie auch im Text selbst so erkennbar gewesen war.

Als Ergebnis der gemeinsamen Textanalyse und vor dem Hintergrund der immanent erfassten Kategorien wurden so zunächst übergeordnete Kategorien herausgearbeitet, die im Folgenden durch eine kursive Schreibweise hervorgehoben werden:

- Bender (1997): Demenz stellt eine individuelle und gesellschaftliche Belastung dar. Diese Belastung muss bewältigt werden. Zwischen Verleugnung und Adaption ermitteln therapeutische Interventionen von außen, die darauf zielen, die erkennbare Beeinträchtigungen bzw. Störungen wie Apathie, Depression und Angst gesellschaftlich zu bewältigen.

- Menne (2002): Die Rekonstruktion der Innenperspektive eines Men- schen mit Demenz verweist auf Prozesse der Anpassung vor allem in der Regulierung von Alltagsaktivitäten. Dieser Prozess ist mit Stress verbunden. Zwischen Abhängigkeit und Unabhängigkeit, dem Bedürfnis nach Bewahrung der Identität und der Erfahrung von Grenzen, dem Bedürfnis, sich selbst ein Wert zu bleiben und für Andere einen Wert zu haben vermitteln ähnliche Vorstellungen darüber, welche Kompetenzen erhaltenswert sind. Die Bewältigungsstrategie ist der Humor.

- Harman (2006): Zwischen Verleugnung/Furcht und der Adaption einer anderen Rolle bewegt sich der Umgang eines Menschen, der dement wird. Forschung hat die Aufgabe, das Krankheitsbild zu beschreiben. Demenz als Krankheit repräsentiert sich durch folgende Erscheinungsformen.

a. Sie ist eine chronische Erkrankung, die in ihrem Verlauf schlimmer wird und ist gekennzeichnet durch einen zunehmenden Verlust von Gedächtnis.

b. Alterungsprozesse befördern die Wahrscheinlichkeit dement zu werden.

c. Sie stigmatisiert.

d. Sie muss bewältigt werden.

- Dechant (2010): Ein Mensch mit Demenz im Frühstadium seiner Erkrankung befindet sich in einem Anpassungsprozess zwischen Abhängigkeit und Unabhängigkeit. Zwischen der Erkenntnis, dass es immer schlimmer wird und nur noch Therapie helfen kann und dem Erleben eines Verlustes, der zu Veränderung zwingt, geht es darum, Kontinuität herzustellen und damit auch das Leben als Herausforderung zu begreifen. Die Identität eines einzelnen Menschen wird durch seine Alltagserfahrung geprägt. Sie zielt auf eine Bewältigung von Angst. Medikamente helfen hier. Die Gesellschaft wiederum empfindet De- 
menz als Krankheitsphänomen und entsprechend auch Menschen, die dement sind, als eine Belastung. Wie bewältigt ein Mensch mit Demenz dieses Problem? Er setzt Kreativität und Humor ein.

Für jeden der exemplarisch ausgewählten vier Texte wurden anschließend immanente Schlüsselkonzepte erfasst. Diese wurden dann in einem dritten Schritt zu einem übergeordneten Modell zusammengefügt. Dazu wurden zunächst gemeinsam in der Autorinnengruppe Matrices erstellt, die in der Rekonstruktion der Textlogik immanente Begründungszusammenhänge widerspiegeln. Es ließ sich definieren, welche Vermittlungsvariablen textimmanent gewählt wurden, um Bezüge zwischen zwei Begriffen zu beschreiben. So wurde in allen vier exemplarisch ausgewählten Texten erkennbar, dass auf der individuellen Seite zwischen Belastung mit und Bewältigung von Demenz Stress als Vermittlungsvariable das beschreibt, was für den pflege- und gesundheitswissenschaftlichen Analyseprozess einen zentralen Forschungsgegenstand darstellt.

Diese Auswertung der immanent herausgearbeiteten Kategorien in einer ebenfalls immanent rekonstruierenden Erfassung von Argumentationsbezügen durch Matrices bildete eine nachvollziehbare Grundlage, um in einem weiteren Schritt die auf beiden Ebenen erkennbaren zentralen Vermittlungsvariablen herausarbeiten zu können. Jede der Kategorien ist im Prinzip in einer direkten Folge auf die entsprechenden Textbezüge zurückzuführen.

Zwei Seiten eines miteinander verbundenen Denkmodells ließen sich im rekonstruierbaren pflege- und gesundheitswissenschaftlichen Blick beschreiben.

- Auf der individuellen Ebene wird erkennbar, dass ein Mensch mit Demenz sich in einem Anpassungsprozess zwischen Abhängigkeit und
Unabhängigkeit befindet. Diese manifestiert sich nach Ansicht der Autorinnen und Autoren vor allem in dem Problem der Bewältigung von Alltagsaktivitäten. Vor dem Hintergrund des Wissens darum, dass es immer schlimmer wird und nur noch Therapie helfen kann, und der Erfahrung eines Verlustes, der zu Veränderung zwingt, geht es auf der positiven Seite darum, Kontinuität herzustellen und damit auch das Leben als Herausforderung zu begreifen. Die Bewältigungsstrategie ist gekennzeichnet durch den Einsatz von Humor und Kreativität.

- Auf der gesellschaftlichen Ebene existieren ähnliche Vorstellungen darüber, welche Kompetenzen erhaltenswert sind. Zwischen individuellen Strategien der Verleugnung, der Adaption einer anderen Rolle und der Kontaminationsvermeidung vermitteln therapeutische Interventionen von außen, die darauf zielen, die erkennbaren Beeinträchtigungen bzw. Störungen wie Apathie, Depression und Angst gesellschaftlich zu bewältigen. Forschung hat die Aufgabe, das Krankheitsbild der Demenz zu beschreiben. Demenz als Krankheit repräsentiert sich durch folgende Erscheinungsformen: Sie ist eine chronische Erkrankung. Sie wird in ihrem Verlauf schlimmer. Sie ist gekennzeichnet durch einen zunehmenden Verlust von Gedächtnis. Alterungsprozesse befördern die Wahrscheinlichkeit, dement zu werden. Sie stigmatisiert. Sie muss bewältigt werden.

\section{Erfassung der Vermittlungsvariablen (Schritt 4)}

Im Rahmen der Modellbildung auf Grundlage der vier zufällig ausgewählten Artikel (vgl. Schritt 3) wurde erkennbar, dass der Begriff der Demenz in beiden Perspektiven im Kontext der Variablen Belastung, Bewältigung und
Stress auftritt. Wie passten nun diese beiden Blickwinkel zusammen? Welche Funktion hatte die Verknüpfung? Demenz wird in jedem Fall als eine $\mathrm{Be}$ lastung bezeichnet, die bewältigt werden muss. Bewältigung wiederum verursacht Stress. Der Alltag als Schaltstelle der Konfrontation zwischen dem Menschen mit Demenz und der Gesellschaft, soll - so die Erkenntnisse aus der systematischen Zufallsauswahl reguliert werden, mit dem Ziel der Eliminierung der Störfaktoren. Der verbindende Begriff ist der der therapeutischen Intervention.

\section{Regelgeleitete Textauswahl (Schritt 5)}

Im Fokus der anschließenden quantitativ ausgerichteten neuen systematischen Suchstrategie standen dann Texte, in denen die drei Vermittlungsvariablen Belastung und/oder Bewältigung und/oder Stress im Kontext Demenz auftauchten. Folgende Begrenzungen wurden ergänzend bei der Textauswahl vorgenommen.

- Es wurden ausschließlich wissenschaftliche Publikationen ausgewählt, die im deutschsprachigen Raum erschienen und auch in deutscher Sprache veröffentlicht worden sind.

- Es wurden mit den Zeitschriften Pflege, Zeitschrift für Gerontologie und Geriatrie und Nervenarzt exemplarisch drei Zeitschriften ausgewählt, in denen Publikationen zu geriatrischen und gerontologischen Themen veröffentlicht werden, um den möglichen dort stattfindenden Diskurs herausarbeiten zu können. Außerdem wurde die wissenschaftliche Literatur, die über das Internetportal googlescholar öffentlich $\mathrm{zu}$ gänglich ist, auf eben jenen aktuellen wissenschaftlichen Diskurs hin untersucht. Eine Begrenzung auf die Jahrgänge 2010 und 2011 fand statt ${ }^{2}$.

- Hinsichtlich der wissenschaftlichen Literatur in der Suchmaschine 
googlescholar galt zusätzlich, dass nur Qualifikationsarbeiten zur Erlangung des Doktortitels (Dissertationen) eingeschlossen wurden, die über das Internet als PDF-Datei frei zugänglich waren. Ausgeschlossen wurden in den Zeitschriften alle Artikel, in denen einer oder mehrere der Schlüsselbegriffe nicht oder nur im Literaturverzeichnis aufgetaucht waren.

Insgesamt wurden fünfzehn relevante Qualifikationsarbeiten und Zeitschriftenartikel gefunden, davon jeweils ein Artikel in der Zeitschrift Pflege sowie in der Zeitschrift für Gerontologie und Geriatrie. In der Zeitschrift Nervenarzt wurden zwei und in der Suchmaschine googlescholar elf Dissertationen gesichtet (Abb. 2).

Erfassung von Argumentationsmustern Die Ergebnisse der Textanalyse der ausgewählten Zeitschriftenartikel und der im Internet über googlescholar frei zugänglichen Dissertationen wurden in der Form von zentralen Argumentationsmustern erfasst. Die Präsentation der Ergebnisse erfolgt in zwei einander ergänzenden Varianten. Zum einen wurden die Argumentationsmuster in den ausgewählten wissenschaftlichen Texten einer immanenten Textanalyse unterzogen. Die Argumentationsmuster, in denen Demenz vor dem Hintergrund der erfassten Vermittlungsvariablen (Belastung, Bewältigung und Stress) erschließbar waren, wurden dazu in einem ersten Schritt in Form von Inhaltsangaben thematisch zusammengestellt und qualitativ hermeneutisch interpretiert. In einer zweiten Variante wurden die gleichen Zusammenfassungen genutzt, um quantitativ herauszuarbeiten, wie oft sich die bereits erfassten Vermittlungsvariablen unter einer individuellen und systemorientierten Perspektive jeweils in welcher Kombination erschließen ließen. Die bereits vorliegenden Vermittlungsvariablen Belas- tung, Bewältigung und Stress wurden dabei um weitere (Unterstützung, Entlastung und Prävention) ergänzt. Diese Ergänzung war notwendig geworden, weil sich in der Analyse diese Begriffe in den ausgewählten Publikationen und Zeitschriftenartikel gezeigt hatte, dass das Argumentationsfeld um Ebenen der Differenzierung erweitert werden musste.

\section{Ergebnisse}

\section{Hermeneutische Textinterpretation}

Folgende Argumentationsmuster konnten identifiziert werden:

- Eine Demenzerkrankung stellt eine hohe Belastung dar. Darin sind sich die Autorinnen und Autoren einig. Sie belastet die Gesellschaft, indem sie hohe Kosten für das Gesundheitssystem verursacht (Günther, 2010; Kalide, 2011; Nordheim, 2011; Ulbrecht 2010).

- Sie belastet die Angehörigen, die sich mit Stigmatisierungsprozessen auseinandersetzen müssen und Schwierigkeiten im sozialen Bezug wie auch in der Organisation des Alltags haben. Sie belastet auch die professionell Pflegenden, die sich mit einer Gruppe von Menschen auseinandersetzen müssen, die zunehmend stärker in ihrer Persönlichkeit verändert erscheinen. Diese Betreuenden leiden nach Ansicht der Autorinnen und Autoren unter diversen Belastungssymptomen. Ihnen muss durch externe Interventionen geholfen werden $\mathrm{Bu}$ scher, 2010; Cramer, 2011; FischerTerworth, 2010; Frey, 2010; Germeroth, 2011; Kalide, 2011; Kurz, 2011; Mantovan, Ausserhofer, Huber, Schulc \& Them, 2010; Nordheim, 2011; Ulbrecht, 2010; Wolf, 2011)

- Letztendlich belastet Demenz auch die Menschen mit Demenz selbst. Diese sollen lernen, sich in die soziale Situation einzubinden und den
Alltag so zu organisieren, dass sie nicht zu einer Belastung für Angehörige und Pflegende werden - das zumindest gilt für die frühe Phase der Erkrankung (Cramer, 2011; Fischer-Terworth, 2010; Leicht, 2011; Perneczky et al., 2011).

Werden diese Argumentationsmuster weiter aufgeschlüsselt, ergibt sich folgendes Bild:

- Der Angehörige ist durch individuelle und passgenaue Interventionen dabei zu unterstützen, die Problemsituation zu bewältigen (Buscher, 2010; Cramer, 2011; Frey, 2010; Germeroth, 2011; Kurz, 2011; Nordheim, 2011; Fischer-Terworth, 2010; Ulbrecht, 2010). Der Mensch mit Demenz ist zwar jemand, der an einer Krankheit leidet, er kommt in diesem Szenario jedoch zumeist lediglich als Verursacher von Belastungen für Andere vor. Es wird auch erkennbar, was einen Menschen mit Demenz am meisten belastet: nicht seine kognitive Einschränkung, sondern seine Verhaltensauffälligkeiten (Wolf, 2011), respektive die Veränderung der $z w i$ schenmenschlichen Verhaltensweisen und der Persönlichkeit (Kurz, 2011; 337). Die Störung der sozialen Interaktion mache das größte Problem aus. Entsprechend werden auch Entlastungen durch möglichst individuell und multimodal wirksame Interventionen für den Angehörigen zu einem zentralen Ziel. Ihre Wirksamkeit gilt es, anhand verschiedener Effektgrößen, zu überprüfen.

- Nordheim (2011) und Fischer-Terworth (2010) sehen ebenfalls beide Personengruppen als belastet an: den von der Krankheit betroffenen Menschen mit Demenz selbst und den pflegenden Angehörigen. Für Nordheim (2011) ist ein Mensch mit Demenz ein Mensch, der in der selbständigen Bewältigung des Krankheitsbildes eingeschränkt sei. Wenn er Unterstützung in Form von nicht 
pharmakologischen Therapien bekäme, dann würde das einen begünstigenden Einfluss auf Kognition und Stimmung sowie alltagspraktische Tätigkeiten haben und letztendlich auch - und damit schließt sich der Kreis der Betroffenheit wieder - die Belastung für die Angehörigen senken.

- Auch Fischer-Terworth (2010) möchte beide Seiten des Belastungsszenarios mit nichtpharmakologischen Interventionen bedienen. Die emotionale Stabilisierung und Stressbewältigung des Angehörigen träfe im gelungenen Fall dann auf einen Menschen mit Demenz, der durch eine Förderung sozialer Interaktion in Form von Psychoedukation, Verhaltenstraining und kognitiver Verhaltenstherapie gelernt hat, sich alltagspraktische und emotionale Bewältigungsstrategien im Umgang mit störungsbedingten Defiziten zu verschaffen. Die Förderung der sozialen Interaktion wäre ein Lösungsmuster, um das als auffällig diagnostizierte Verhalten des Menschen mit Demenz und die Belastung des Angehörigen im Umgang mit diesem persönlichkeitsveränderten Menschen wieder in ein Gleichgewicht $\mathrm{zu}$ bringen. Die gesellschaftliche Intervention versuche über eine therapeutisch ausgerichtete Intervention also, den als hoch bezeichneten Leidensdruck des Angehörigen zu reduzieren, damit der Mensch mit Demenz so lange wie möglich in der häuslichen Umgebung bleiben kann. Da die Versorgung von Menschen mit Demenz zudem hohe gesellschaftliche Kosten verursacht, ist diese Lösung zudem zumindest aktuell die (scheinbar) preiswertere Variante für das Gesundheitssystem, wäre da nicht das zusätzliche Problem, dass auch die Angehörigen durch ihre Be- lastung dem Gesundheitssystem hinsichtlich Extrakosten zur Last fallen (können).

- Wenn der Blick direkt auf den Menschen mit Demenz selbst fällt, dann geht es darum, wie sich in der Konfrontation mit der Demenz für den Betroffenen das Selbstbild ändert und wie sich eine selbsterhaltende Reaktion von einem vollständigen Fehlen der Krankheitseinsicht unterscheidet (Leicht, 2011). Nach Leicht erfasst Clare eine individuelle Bewältigungsstrategie des Menschen mit beginnender Demenz, die sich auf dem Kontinuum von Selbsterhaltung und Selbstanpassung ergibt, und grenzt sie gegenüber der Anosognosie ab, in der der Patient ein vollständiges Fehlen von Krankheitseinsicht zeige. Krankheitsverdrängung wird so auch als eine mögliche und nicht unwirksame Strategie der Bewältigung von Demenz bzw. ihrer

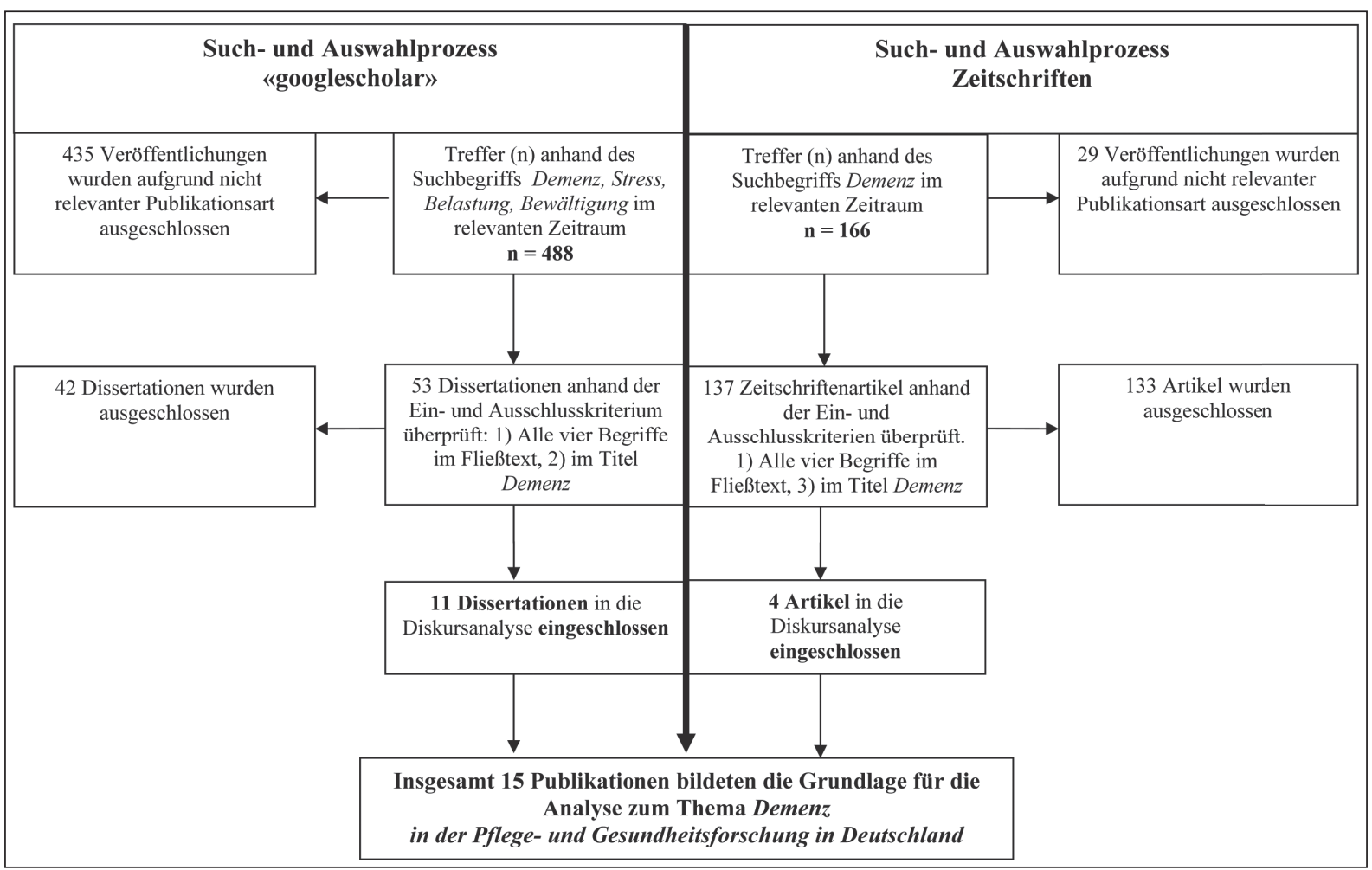

Abbildung 2: Darstellung der regelgeleiteten Textauswahl intus Ein- und Ausschlusskriterien (Schritt 5). 
Integration in das eigene Selbstbild gesehen (Leicht, 2011).

Werden diese in den 15 ausgewählten pflege- und gesundheitswissenschaftlichen Artikeln bzw. Dissertationen vertretenen Positionen zusammengefasst, dann ergibt sich folgendes Bild: Eine Demenzerkrankung stellt für die Gesellschaft, die pflegenden Angehörigen und andere Betreuungspersonen eine belastende Herausforderung dar. Sie zwingt die Gesellschaft zu einer Auseinandersetzung mit diesem Krankheitsbild, das die Persönlichkeitsveränderung zu einem sozialen Problem macht. Sie hat hohe Folgekosten, weil auch die pflegenden Angehörigen durch ihre Belastungen krank werden können. Die Gesellschaft sorgt durch geeignete Interventionen dafür, dass Menschen mit Demenz so lange wie möglich in ihrem häuslichen Umfeld bleiben können.

Demenz ist auf der individuellen Ebene vor allem eine Belastung für die Angehörigen. Sie werden als gestresst bezeichnet. Ihnen steht Unterstützung zu. Sie sind vornehmlich diejenigen, die Demenz bewältigen müssen. Dabei ist es weniger die kognitive Beeinträchtigung, die Stress auslöst, sondern die Veränderung der Persönlichkeit des Menschen mit Demenz durch Verhaltensauffälligkeiten, respektive die Veränderung der zwischenmenschlichen Verhaltensweisen und der Persönlichkeit, die Probleme erzeugt. Die soziale Integration in der Form, dass Angehörige und Menschen mit Demenz wieder besser miteinander umgehen können, ist das Ziel der als therapeutisch bezeichneten Interventionen. Menschen können sich durch einen intellektuellförderlichen Lebensstil in einem gewissen Grad vor Demenz schützen. Wenn sie selbst von Demenz betroffen sind, entscheidet ihr Resilienzvermögen darüber, wie sie damit umgehen können. Krankheitsverdrängung ist in diesem Sinne auch eine mögliche Be- wältigungsstrategie, wenn sie zu einer Integration der Krankheit in das Selbstbild führen kann.

\section{Kombinationen und Häufigkeiten}

Das Thema Demenz in der Kombination mit den Vermittlungsvariablen Belastung, Stress und Bewältigung respektive in der Ergänzung Unterstützung, Entlastung und Prävention wird in den analysierten pflege- und gesundheitswissenschaftlichen Texten auf zwei Ebenen diskutiert: zum einen auf der Ebene des Individuums unter den drei Perspektiven Angehörige von Menschen mit Demenz, Menschen mit Demenz und professionell Betreuende, und zum andern auf der Ebene von Systemkomponenten unter den drei Perspektiven Gesellschaft, Wirtschaft und Soziales. Als zentral erweist sich für jede der Perspektiven die Frage, wie mit den hohen persönlichen und wirtschaftlichen, gesellschaftlichen und sozialen Kosten der Demenz umzugehen ist.

Folgendes Ergebnis zeichnet sich ab:

- Belastung: Durch die Demenz belastet sind vor allem die Angehörigen von Menschen mit Demenz (12 Nennungen), gefolgt von den Menschen mit Demenz selbst (8 Nennungen) und den professionell Betreuenden (3 Nennungen). Die Demenz verursacht für die beiden erstgenannten Gruppen hohe persönliche $\langle$ Kosten〉. Belastet ist aber auch das gesellschaftliche (5 Nennungen) und wirtschaftliche System (4 Nennungen). Die Gruppe, die in den ausgewählten wissenschaftlichen Artikeln als am meisten belastet erscheint, sind die Angehörigen.

- Bewältigung: Es werden nur auf der persönlichen Ebene die Angehörigen von Menschen mit Demenz (5 Nennungen) und Menschen mit Demenz selbst (5 Nennungen) benannt.

- Unterstützung: benötigen auf der persönlichen Ebene die Angehörigen von Menschen mit Demenz (8
Nennungen) und Menschen mit Demenz selbst (4 Nennungen). Es ist auffällig, dass in diesem Punkt die Angehörigen mehr als doppelt so häufig als unterstützungswürdige Personen benannt werden. Die Systemperspektive findet an dieser Stelle keine Beachtung.

- Entlastung: hier werden die Gesellschaft (4 Nennungen) und die Wirtschaft (2 Nennungen) angeführt.

- Prävention: Präventive Maßnahmen werden dreimalig für den Bereich des Sozialen (z. B. Lebensstil) angesprochen.

Aus der rein summarischen Aufzählung (Tab. 1) geht hervor, dass es vor allem die Angehörigen sind, die als belastet angesehen werden. Sie sollen auf der persönlichen Ebene unterstützt werden. Menschen mit Demenz werden weniger häufig als persönlich Belastete benannt. Sie sind in einem signifikant geringeren Umfang auch weniger die Personen, die Unterstützung auf der individuellen Ebene bekommen und erfahren. Belastet wird unter einem Systemaspekt die Gesellschaft durch hohe Kosten. Prävention wiederum wird ebenfalls auf der sozialen, also Systemebene eher selten benannt.

\section{Diskussion}

\section{Das methodische Verfahren}

Das eingesetzte methodische Verfahren zur Erschließung eines wissenschaftlichen Diskurses zum Thema Demenz hat sich als grundsätzlich tragfähig erwiesen. Die Exemplarik eines Diskursmodells zunächst einmal qualitativ herauszuarbeiten, um dieses dann im Rahmen einer regelgeleitet angelegten quantitativen Literaturrecherche einzusetzen, ermöglichte es, größere Datenmengen im Rahmen einer Inhaltsanalyse zu erschließen. Es handelt sich um eine Form der integra- 


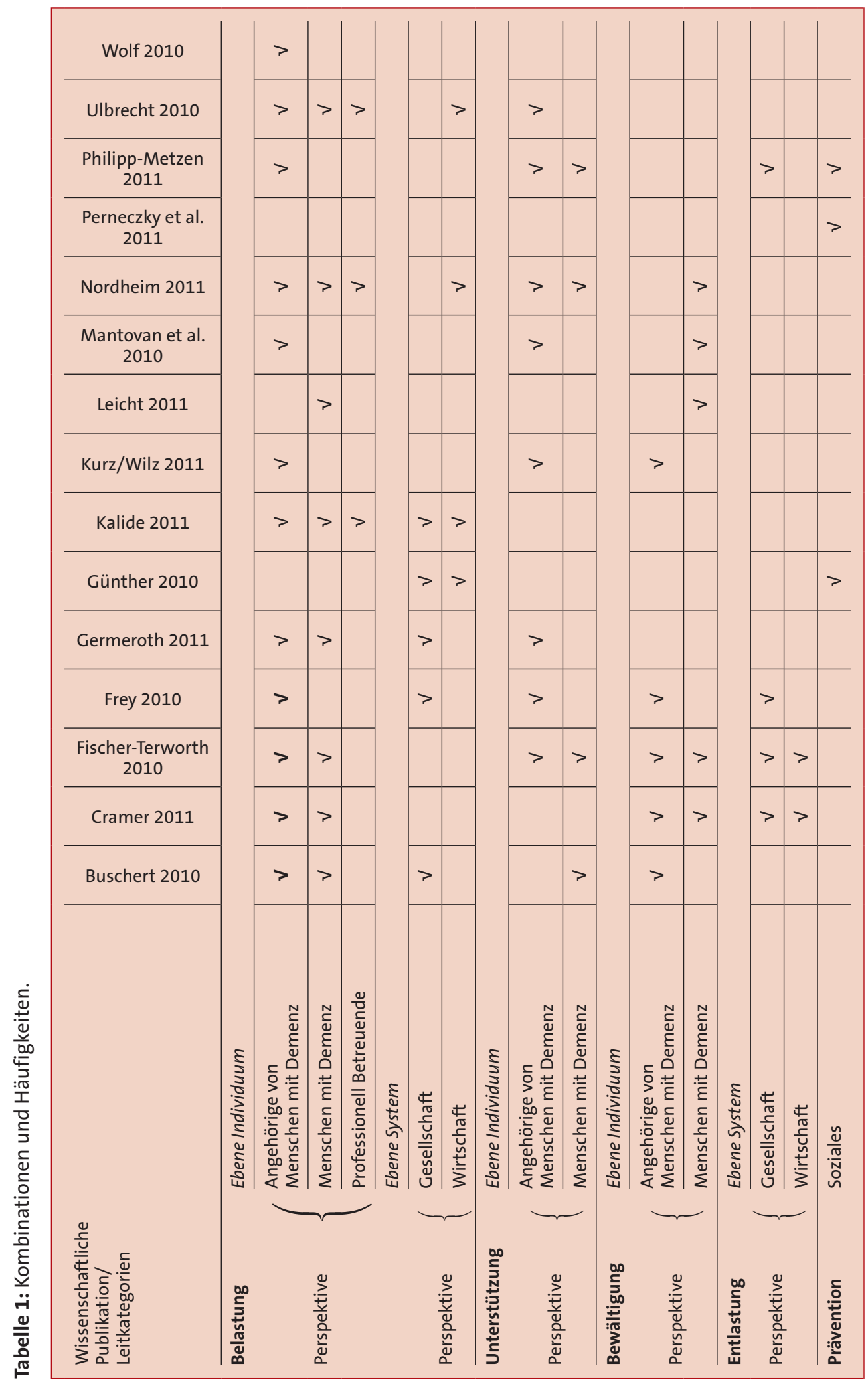


tiven Methodenkombination (vgl. Kelle, 2008).

Folgende Einschränkungen sind zu benennen, die in einer Folgeanalyse beachtet und mit neuen Lösungsansätzen konfrontiert werden müssen:

- Die Entwicklung von Vermittlungsvariablen als Rekonstruktion von immanenten Argumentationsmustern mithilfe der Software MAXQDA ist möglich. Der Arbeitsaufwand war zwar vergleichsweise hoch, aber dadurch zu rechtfertigen, dass die erschlossenen Variablen für die anschließende zweite Phase der Literaturrecherche genutzt werden konnten.

- Problematisch erschien es im Nachhinein, dass einige der exemplarisch ausgewählten Texte englischsprachig waren. Es entstanden Übersetzungsprobleme. In weiteren Analysen sollte eine Auswahl der Texte für beide Analyseverfahren in der gleichen Sprache erfolgen. Eine Erfassung des internationalen Diskurses könnte im Anschluss daran folgen.

- Die zeitliche Begrenzung der Literaturauswahl auf die Jahre 2010 und 2011 war aus arbeitstechnischen Gründen für die ersten vier Schritte der Auswertung berechtigt und sinnvoll. In einer Folgestudie wäre allerdings zu überlegen, ob der Erhebungszeitraum für das gewählte Thema auszuweiten ist.

- Das methodische Verfahren zur Erarbeitung der Vermittlungsvariablen hat sich generell als sinnvoll, allerdings an einem Punkt auch als auch störanfällig erwiesen. Im Schritt 6 der Analyse (Erfassung von Argumentationsmustern) mussten die herausgearbeiteten Vermittlungsvariablen erweitert werden. Diese Anpassung verweist vermutlich auch auf eine methodische Schwachstelle im dritten Schritt der Analyse (Reduktion und Modellbildung). Es wäre zu überlegen, ob in einer Fol- gestudie in dem ersten Schritt der Analyse (Exemplarische Textauswahl) entsprechend dem methodologischen Vorgehen der Grounded Theory das Sample der immanenten Kategorienbildung im Sinne eines Theoretischen Samplings gebildet wird (vgl. Glaser \& Strauss, 2010 61 -65), bevor die gewonnenen Variablen als Grundlage für eine weitere Literaturrecherche (Schritt 5) benutzt werden. Damit könnte die Störanfälligkeit zumindest im Sinne der qualitativen Forschungsmethode möglicherweise eingegrenzt werden. Dementsprechend erscheint es auch geraten, die quantitative Analyse um eine Analyse von Texten mit Antonymen der Vermittlungsvariablen zu ergänzen.

\section{Die inhaltliche Dimension}

Der dritte Schritt der integrativen Analyse führte vor dem Hintergrund einer exemplarischen immanenten Textanalyse zur Bildung von Schlüsselkonzepten. Diese waren grundlegend für eine Modellbildung. Die Modellbildung wiederum war eine notwendige Voraussetzung für die Bestimmung von Vermittlungsvariablen (Schritt 4). Es ließ sich in einer regelgeleiteten Textanalyse auf zwei Wegen (qualitative hermeneutische Textanalyse und quantitative Erfassung von Häufigkeiten) herausarbeiten, dass Demenz sowohl auf der individuellen als auch auf der gesellschaftlichen Ebene mit den Vermittlungsvariablen Belastung, Stress und Bewältigung argumentativ verbunden ist.

Ein eindeutiges Ergebnis wurde erkennbar: Als eigentlich Belastete werden vor allem die Personen bezeichnet, die Menschen mit Demenz betreuen, allen voran die Angehörigen. Ihnen gilt die gesellschaftliche Unterstützung. Zwischen dem Menschen mit Demenz und der Gesellschaft befindet sich der Angehörige als derjenige, der mit der
Demenz umgehen muss. Die Belastung für Angehörige entsteht, weil sie diejenigen sind, die sich in dem sozialen Bezugsfeld mit Menschen mit Demenz mit der Veränderung der Persönlichkeitsstruktur derselben auseinandersetzen müssen. Nicht die kognitive Beeinträchtigung erzeugt die größten Belastungen für die Angehörigen, sondern die Persönlichkeitsveränderungen.

Wesentlich ist es nun, und damit werden bereits Lösungsmuster anvisiert, dass die sozialen Beziehungen zwischen Angehörigem und Menschen mit Demenz vor allem auch in Bezug auf die Organisation des Alltags so geregelt werden, dass keine Störungen nach außen auftreten. Wenn diese Störungen allerdings vorhanden sind, dann tragen auch nichtmedikamentöse Therapien dazu bei, die soziale Interaktion zwischen Menschen mit Demenz und ihren Angehörigen zu verbessern. Wie Menschen mit Demenz ihre Erkrankung in ihr Selbstbild integrieren und Angehörige damit umgehen können, hängt, folgt man dem immanenten Argumentationsmuster, auch von ihrem jeweiligen Resilienzvermögen ab. Perspektivisch impliziert dies die Möglichkeit von kreativen Lösungen, zu denen allerdings noch geforscht werden muss. Wie ein Mensch mit Demenz seine Erkrankung in sein Selbstbild integrieren kann, also Demenz bewältigt, ist unter dieser Perspektive eine Frage seiner eigenen Ressourcen und der Hilfestellung, die er von außen bekommt. Therapeutische Interventionen dienen vor allem dazu, dass er in die Lage versetzt wird, den Alltag angemessen zu bewältigen. Damit trägt er zu einer Entlastung der Betreuungspersonen bei.

\section{Perspektiven}

Überprüft man die zentralen inhaltlichen Schlussfolgerungen noch einmal daraufhin, welche konkreten Perspek- 
tiven sie für die Pflege- und Gesundheitsforschung eröffnen, lässt sich folgendes festhalten:

An der zugewiesenen gesellschaftlichen Funktion, Demenz als Metapher für eine krankheitsbedingte Unvernunft zu sehen, hat sich, betrachtet man den rekonstruierten Diskurs zum Umgang der Pflege- und Gesundheitsforschung mit Menschen mit Demenz, eigentlich erstaunlich wenig geändert.

Es ist erkennbar - und damit lässt sich zumindest eine Idee davon erahnen, warum Demenz immer noch der Seite des Wahnsinns bzw. der Geisteskrankheiten zugeordnet wird - dass die größte Belastung, die für die Angehörigen herausgearbeitet wird, nicht so sehr die kognitiven Beeinträchtigungen, sondern eher die Persönlichkeitsveränderungen generell sind. Das bringt die interessante Konstruktion eines Handlungsmodells hervor, in der der Angehörige/die Angehörige fast gemeinsam mit dem Menschen mit Demenz in eine eigendynamische $\mathrm{Au}$ ßenseiterrolle gerät. In einen als persönlich definierten Rahmen gesetzt, sorgen diese Interaktionspartnerinnen und -partner dafür, dass die Gesellschaft von der Konfrontation mit diesem Problem weitgehend befreit bleibt. So wird dem zunehmend unsichtbar werdenden Menschen mit Demenz ein Wesen namens Angehöriger an die Seite gestellt. Diesem wird die Aufgabe der Bewältigung zugeteilt. Dabei wird ihm von der gesellschaftlichen Seite aus Unterstützung dann zugesichert, wenn er das Problem des Menschen mit Demenz kompensieren kann.

Der Umgang mit Menschen mit Demenz in dem rekonstruierten wissenschaftlichen Diskurs verweist also auf zwei Seiten dieses Diskurses, die einander ergänzen: auf der einen Seite wird ein Mensch mit Demenz zu einem Wesen, das eine für andere belastende Persönlichkeitsveränderung durchmacht. Gemeinsam mit seinem versorgenden
Angehörigen wird er in eine Außenseiterstellung versetzt. Auf der anderen Seite werden die kognitiven Beeinträchtigungen, die ihm als Krankheitsbild zugewiesen werden, für den $\mathrm{Au}$ ßenstehenden zu einem Symbol der Hoffnung auf Kreativität, die genau in diesem Verlust von Vernunft verankert ist.

Auf den Begriff des Diskurses im Sinne von Foucault selbst rekurriert, würde sich nun die Frage nach den praktischen Konsequenzen dieses rekonstruierten Diskursmodells für die Wissenschaft gar nicht stellen. Die Funktionsweise von Machtstrukturen $\mathrm{zu}$ entschlüsseln, ist nicht unmittelbar mit der Perspektive verbunden, diese auch verändern zu können. Das Muster der Ausgrenzung der Dyade Angehöriger und Mensch mit Demenz aus der gesellschaftlichen Teilhabe als zentrales Diskurselement sagt so zunächst einmal nicht zwingend etwas darüber aus, wie man diesen Mechanismus auflösen kann. Und es sagt auch nichts darüber aus, was die in diesen Diskurs involvierten konkreten Personen erleben. Aber genau das wäre ein möglicher Ansatzpunkt, um diesen Diskursmechanismus aufzubrechen: den von Demenz betroffenen Menschen selbst in der Forschung deutlicher sichtbar zu machen, seine Bedarfe und Bedürfnisse zu rekonstruieren, seine Individualität und seine eigenen Strategien der Bewältigung zu erfassen, eröffnet zumindest eine Möglichkeit, den geschilderten Ausgrenzungsprozessen entgegenzuwirken.

Um in dieser Perspektive weiterdenken zu können wäre zunächst einmal die eingangs geschilderte auch methodische Differenz zwischen Diskursanalyse und Beschreibung phänomenologischen Typs oder anderer interpretativer Methoden produktiv aufzunehmen. Interpretative Methoden greifen einen anderen Aspekt und eine andere Sichtweise der Analyse auf. Es er- scheint sinnvoll, sowohl den kritisch strukturierenden Blick der Diskursanalyse und damit die Analyse von gesellschaftlichen Machtstrukturen als auch den eher von innen rekonstruierenden Blick auf die Muster der Handlungslogik individueller Personen zu nutzen, um den Blick auf mögliche Veränderungspotenziale in einer aktuellen Handlungspraxis zu richten. Die Erkenntnisse, die sich aus einer phänomenologischen Sichtweise ergeben und die Erkenntnisse einer Diskursanalyse könnten sich in der Bewältigung ganz konkreter Handlungssituationen insofern verbinden, als sie zwei einander ergänzende Blickwinkel darstellen. Der scheinbare Widerspruch, der dabei z.B. zwischen einem Modell der Fürsorge als Machtstruktur und einer ganz konkreten situativen Handlungslogik liegt, kann auch produktiv genutzt werden. Dass es dabei immer auch darum geht, die gesellschaftlichen Rahmenbedingungen, die familiäre Situation und den aktuellen Status des Umgangs der Gesellschaft mit Menschen mit Demenz sowie weitere Einflussfaktoren zu entschlüsseln, ist notwendig, um die Möglichkeiten, die ein Versorgungsnetz bietet, auch angemessen erschließen zu können: im Sinne und für den von Demenz betroffenen Menschen und seinen Angehörigen, aber auch für den, der diese Menschen professionell auf ihrem Weg der Bewältigung begleitet. In diesem Feld der Forschung stellen sich Herausforderungen für die Zukunft. Den Diskurs der Demenz zu rekonstruieren, ist dabei nur ein Baustein.

\section{Beiträge der einzelnen Autorinnen}

Datenerhebung: BPK, OK und GK

Datenmanagement: BPK, OK und GK

Dateninterpretation: BPK, OK und GK Verfassen des Manuskripts: BPK, OK und GK 
The scientific discourse about

dementia in Germany - first results of the exemplary exercise of an integrative methodological approach

Background: The analysis of the discourse in dealing with people with dementia within the nursing and health care research in Germany is previously missing.

Aim: How are people with dementia described in the discourse of the German nursing and health care research community and what does that mean for the nursing and health research in Germany?

Method: Using an integrative methodological approach, the first steps of an analysis of dementia discourse are developed and exemplarily tested in a selected limited contextual framework. Result: The scientific discourse is focused on the burden of the family caregivers of the person with dementia. They are identified as those who have to handle the burden with their spouse or siblings with dementia.

Conclusions: A methodological approach of integrative analysis was tested. According perspectives should be developed to incorporate the perspective of people with dementia in a stronger way.

Key words: discourse, dementia, integrative methodological approach, key concepts, mediation variables

\section{Literatur}

Bender, M. P.; Cheston, R. (1997). Inhabitants of a Lost Kingdom: A Model of the Subjective Experiences of Dementia. Aging and Society, 17 (5), 513-532.

Bohnsack, R.; Marotzki, W.; Meuser, M. (Hrsg.). (2003). Hauptbegriffe Qualita- tiver Sozialforschung. Opladen: Leske \& Budrich.

Buschert, V. C. (2010). Entwicklung einer kognitiven Intervention bei amnestischerleichter kognitiver Störung (LKS) und leichtgradiger Alzheimer-Demenz (AD) - Evaluierung auf neuropsychologischer und neurobiologischer Ebene unter Berücksichtigung des stadienspezifischen Ansatzes. Dissertation zum Erwerb des Doktorgrades der Humanbiologie an der Medizinischen Fakultät der Ludwig-Maximilians-Universität München. http://edoc.ub uni-muenchen.de/12289/1/Buschert Verena.pdf [26.6.2012].

Cramer, B. (2011). Biografiearbeit und kognitive Verhaltenstherapie bei Alzheimer Krankheit im Frühstadium. Eine multimethodische und multiperspektivische Untersuchung mit Patienten, Angehörigen und Psychotherapeuten. Inaugural - Dissertation zur Erlangung des Doktorgrades der Philosophie an der Fakultät für Psychologie und Pädagogik der Ludwig-Maximilians-Universität München. edoc.ub.uni-muenchen. de/13465/1/Cramer_Barbara.pdf [26.6.2012].

Dechant, R.; Zimmermann, C.; Merlin, H (2010). «Ich bin ja noch ich». Rita Dechant, Christian Zimmermann und Helen Merlin über Ihren Alltag als Demenzkranke, ihre Strategie beim Outing und ihren Umgang mit dem allmählichen Verlust der Selbständigkeit. Spiegel Wissen, 1, 34-41.

Fischer-Terworth, C. (2010). Evaluation einer TEACCH-basierten psychologischen Intervention bei leichter bis mittlerer Demenz: Eine kontrollierte Studie. Dissertation zur Erlangung der Würde des Doktors der Philosophie im Fach Psychologie an der Universität Hamburg. http://ediss.sub.uni-hamburg.de/volltexte/2010/4561/pdf/Fischer-Terworth-dissertation.pdf [26.6.2012].

Foucault, M. (1969). Wahnsinn und Gesellschaft. Eine Geschichte des Wahns im Zeitalter der Vernunft. Frankfurt am Main: Suhrkamp.

Foucault, M. (2009). Geometrie des Verfahrens. Schriften zur Methode. Frankfurt am Main: Suhrkamp.
Frey, C. (2010). Helfer, Hilfen und Demenz. Angehörige Demenzkranker im Kontext formeller und informeller Hilfen. Eine empirische Studie. InauguralDissertation zur Erlangung des Doktorgrades der PhilosophischPädagogischen Fakultät der Katholischen Universität Eichstätt-Ingolstadt. http://www.opus-bayern.de/ ku-eichstaett/volltexte/2011/83/pdf/ Diss_Frey_2010_aktuellste.pdf [26.6.2012].

Germeroth, S. A. (2011). Auswirkungen von Nutzung und Nicht-Nutzung einer vom Hausarzt vermittelten zugehenden Form der Angehörigenberatung bei zu Hause versorgten Patienten mit leichter und mittelschwerer Demenz. InauguralDissertation zur Erlangung der Doktorwürde der Medizinischen Fakultät der Friedrich-Alexander-Universität Erlangen-Nürnberg.http://www.opus. u..unierlangen.de/opus/volltexte/2011/2288/ pdf/Stefanie_Germeroth_Dissertation. pdf [26.6.2012].

Glaser, B. G.; Strauss, A. L. (2010). Grounded Theory. Strategien qualitativer Forschung. Bern: Hans Huber.

Günther, F. (2010). Homocystein, Liquorparameter und Apolipoprotein E bei Demenzerkrankungen und leichter kognitiver Störung. Inaugural-Dissertation zur Erlangung der Doktorwürde der Medizinischen Fakultät der FriedrichAlexander-Universität Erlangen-Nürnberg. http://www.opus. u..uni-erlangen. de/opus/volltexte/2010/2000/pdf/ D I S S E R T A T I O N.ge s a m t . p d f [26.6.2012].

Harman, G.; Clare, L. (2006). Illness Representations and Lived Experience in Early-Stage Dementia. Qualitative Health Research, 16 (4) 484-502.

Kalide, J. (2011). Die Bedeutung demenzieller Erkrankungen in der Medizin für das Individuum und die Gesellschaft. Studie im Quer- und Längsschnitt. Dissertation zur Erlangung des Doktorgrades an der Medizinischen Fakultät. Charité - Universitätsmedizin Berlin. http://www.diss.fu-berlin.de/diss/servlets/MCRFileNodeServlet/FUDISS_derivate_000000012067/Aus_der_Klinik_ fuer_Altersmedizin_und_Fruehrehabilitation.pdf?hosts $=$ [26.6.2012]. 
Kelle, U. (2008). Die Integration qualitativer und quantitativer Methoden in der empirischen Sozialforschung. Wiesbaden: VS Verlag für Sozialwissenschaften.

Kuckartz, U. (2007). Einführung in die computergestützte Analyse qualitativer Daten. Wiesbaden: VS Verlag für Sozialwissenschaften.

Kuckartz, U. (2012). Qualitative Inhaltsanalyse. Methoden, Praxis, Computerunterstützung. Weinheim und Basel: Juventa Beltz.

Kurz, A.; Wilz, G. (2011). Die Belastung pflegender Angehöriger bei Demenz. Entstehungsbedingungen und Interventionsmöglichkeiten. Nervenarzt, 82 (3), $336-342$.

Leicht, H. (2011). Fehlende Krankheitseinsicht bei Alzheimer-Demenz und methodische Aspekte ihrer Erfassung. Kumulative Dissertation zur Erlangung des Doktorgrades an der Medizinischen Fakultät der Universität Leipzig. http://www.qucosa.de/fileadmin/ data/qucosa/documents/6791/Dissertation_HLeicht_2011.pdf [26.6.2012].

Mantovan, F.; Ausserhofer, D.; Huber, M.; Schulc, E.; Them, C. (2010). Interventionen und deren Effekte auf pflegende Angehörige von Menschen mit Demenz - Eine systematische Literaturübersicht. Pflege 23 (4), 223 - 239.

Mayring, P. (2007). Qualitative Inhaltsanalyse. Grundlagen und Techniken. Weinheim und Basel: Beltz.

Menne, H. L.; Kinney, J. M.; Morhardt, D. J. (2002). 'Trying to continue to do as much as they can do'. Theoretical insights regarding continuity and meaning making in the face of dementia. Dementia, 1 (3), 367 - 382.

Nordheim, J. (2011). Gesundheitliche Versorgung älterer Menschen mit Demenz: Eine Sekundäranalyse auf der Basis von Krankenkassenroutinedaten. Dissertation zur Erlangung des Doktorgrades an der Medizinischen Fakultät Charité - Universitätsmedizin Berlin. http://www.diss.fu-berlin. de/diss/servlets/MCRFileNodeServlet/FUDISS_derivate_000000009361/ JNordheim_Diss_eVer\%C3\%B6ffentl. pdf?hosts [26.6.2012].
Perneczky, R.; Alexopiulos, P.; Schmidt, G.; Sorg, G.; Förstl, H.; Diehl-Schmid, J.; Kurz, A. (2011). Kognitive Reservekapazität und ihre Bedeutung für Auftreten und Verlauf der Demenz. Nervenarzt, 82 (3), 325-335.

Philipp-Metzen, H. E. (2011). Die Enkelgeneration in der familialen Pflege bei Demenz Erfahrungen und Bilanzierungen - Ergebnisse einer lebensweltorientierten Studie. Zeitschrift für Gerontologie und Geriatrie, 44 (6), 397 - 404.

Ulbrecht, G. (2010). Qualitätsziele angehörigenentlastender und -unterstützender Dienste für zuhause lebende Demenzkranke aus Anbieter und Expertenperspektive. Inaugural-Dissertation zur Erlangung der Doktorwürde an der Medizinischen Fakultät der FriedrichAlexander-Universität Erlangen-Nürnberg. http://www.opus. u..uni-erlangen. de/opus/volltexte/2011/2257/pdf/ GudrunUlbrechtDissertation.pdf [26.6.2012].

Wolf, S. A. (2011). Einfluss von Acetylcholinesterase-Inhibitoren auf neuropsychologische Leistungen von Patienten mit Demenz vom Alzheimer-Typ. Dissertation zur Erlangung des Doktorgrades an der Fakultät für Naturwissenschaften der Otto-von-GuerickeUniversität Magdeburg.http://edoc. bibliothek.uni-halle.de/servlets/MCRFil e Nod e S ervlet / H A L C o Re_ derivate_00004792/S._A._Wolf_Einfluss_von_AChEI_auf_neuropsychologische_Leistungen_von_Patienten_ mit_DAT.pdf [26.6.2012].

Wrana, D.; Ziem, A.; Reisig, M.; Nonhoff, M.; Angermüller, J. (2014). DiskursNetz. Wörterbuch der interdisziplinären Diskursforschung. Berlin: Suhrkamp.
Was war die größte Herausforderung bei Ihrer Studie?

Die Entwicklung eines methodischen Ansatzes zur Analyse von großen Datenmengen im Rahmen der Diskursanalyse zum Thema Demenz.

Was wünschen Sie sich bezüglich der Thematik für die Zukunft?

Die Analyse des wissenschaftlichen Diskurses zum Thema Demenz wird fortgesetzt und trägt zu einer kritischen Reflexion der Forschung bei.

Was empfehlen Sie den LeserInnen zum Weiterlesen/Vertiefen? Wrana et al. (2014). DiskursNetz. Wörterbuch der interdisziplinären Diskursforschung. Berlin: Suhrkamp.

\section{Korrespondenzadresse}

PD Dr. Birgit Panke-Kochinke Arbeitsgruppe Methoden in der Versorgungsforschung (METH) Deutsches Zentrum für Neurodegenerative Erkrankungen Postfach 6250
Stockumer Straße 12 58453 Witten, Deutschland Tel.: +49 2302 926-227 Fax: +49 $2302926-239$

birgit.panke-kochinke@dzne.de 